\title{
Stochastic Gravitational Wave Background from $\mathrm{PBH}-\mathrm{ABH}$ Mergers
}

\author{
Wenfeng Cui (崔文峰), ${ }^{a}$ Fei Huang (黄飞), ${ }^{a, b}$ Jing Shu (舒 \\ 菁), ${ }^{a, c, d, e, f, g}$ and Yue Zhao (赵悦) ${ }^{h}$
}

\author{
${ }^{a}$ CAS Key Laboratory of Theoretical Physics, Institute of Theoretical Physics, \\ Chinese Academy of Sciences, Beijing 100190, China \\ ${ }^{b}$ Department of Physics and Astronomy, University of California, Irvine, CA 92697 USA \\ ${ }^{c}$ School of Physical Sciences, University of Chinese Academy of Sciences, \\ Beijing 100049, China \\ ${ }^{d}$ CAS Center for Excellence in Particle Physics, Beijing 100049, China \\ ${ }^{e}$ Center for High Energy Physics, Peking University, Beijing 100871, China \\ ${ }^{f}$ School of Fundamental Physics and Mathematical Sciences, \\ Hangzhou Institute for Advanced Study, UCAS, Hangzhou 310024, China \\ ${ }^{g}$ International Centre for Theoretical Physics Asia-Pacific, Beijing/Hangzhou, China \\ ${ }^{h}$ Department of Physics and Astronomy, University of Utah, Salt Lake City, UT 84112, USA \\ E-mail: cuiwenfeng@itp.ac.cn, huangf4@uci.edu, jshu@itp.ac.cn, \\ zhaoyue@physics.utah.edu
}

\begin{abstract}
The measurement of gravitational waves produced by binary black-hole mergers at the Advanced LIGO has encouraged extensive studies on the stochastic gravitational wave background. Recent studies have focused on gravitational wave sources made of the same species, such as mergers from binary primordial black holes or those from binary astrophysical black holes. In this paper, we study a new possibility - the stochastic gravitational wave background produced by mergers of one primordial black hole and one astrophysical black hole. Such systems are necessarily present if primordial black holes exist. We study the isotropic gravitational wave background produced through the history of the Universe. We find it is very challenging to detect such a signal. We also demonstrate that it is improper to treat the gravitational waves produced by such binaries in the Milky Way as a directional stochastic background, due to a very low binary formation rate.
\end{abstract}




\section{Contents}

1 Introduction 1

2 Isotropic Cosmological Background 2

2.1 GW power spectral density 2

2.2 GW radiation power spectrum 3

2.3 Merger Rate 3

2.3.1 PBH distribution $\quad 4$

2.3.2 ABH distribution 5

2.3.3 ABH-PBH binary formation probability 6

2.3.4 Halo mass function 8

2.4 Isotropic SGWB power spectral density from ABH-PBH merger 8

3 PBH-ABH binaries in the Milky Way 9

4 Conclusion

\section{Introduction}

The first detection of gravitational waves (GW) [1] by the LIGO and Virgo collaborations $[2,3]$ in 2015 has opened a new window to study astrophysics and cosmology. Since then, many compact binary coalescence events have been observed, including mergers of binary black holes, binary neutron stars and black hole-neutron star binaries [4-6]. Due to limited sensitivities, those events are located at relatively low redshift, i.e., $z \lesssim 1$. Meanwhile, binary mergers can occur at a much earlier time. Binary mergers of astrophysical black holes (ABHs) can occur shortly after the formation of first stars. Primordial black holes (PBHs) can be produced due to large density perturbations in the early Universe. The mergers of PBHs could even start deeply within the radiation-dominated epoch and through almost the entire history of the Universe.

For an individual GW source at a large redshift, the signal is too weak to be detectable. However, the incoherent superposition of a large number of unresolved sources may constitute an observable stochastic gravitational background (SGWB). The detection or the absence of such a background can therefore teach us about the properties of the GW sources. For example, the null detection of the SGWB produced by ABH-ABH mergers can be used to constrain various formation scenarios of binary ABHs [7]. Similarly, the search of SGWB produced by PBH-PBH mergers can also be exploited to study the fraction of dark matter in the form of PBHs $[8,9]$. In addition, when combined with the merger-rate history, the SGWB can also be used to distinguish between the population of ABHs from that of PBHs [10-13].

Besides binary mergers, many other sources can also produce the SGWB, for example, astrophysical sources like supernovae [14-17] and magnetars [18-22], and cosmological sources such as cosmic string [23], inflation [24-27] and first-order phase transitions [28, 29]. For different types of sources, their characteristic frequency as well as the spectral shape can be very different.Therefore, various species of GW experiments are needed in order to explore interesting physics in different frequency bands. 
Typically, ground-based interferometers have sensitivities at relatively high frequency domain. For example, LIGO, Virgo and KAGRA aim for signals with frequencies between $\sim 10-10^{3} \mathrm{~Hz}$. On the other hand, space-based GW experiments can observe GW at a much lower frequency. For instance, LISA, Taiji and TianQin [30-33], have the optimal sensitivities from $10^{-4} \mathrm{~Hz}$ to $10^{-1} \mathrm{~Hz}$. Further lower frequency gravitational waves can be searched by pulsar timing arrays [34, 35]. Currently, Advanced LIGO and Advanced Virgo have placed an upper limit on the dimensionless GW energy density, $\Omega_{\mathrm{GW}}$, for isotropic background at approximately $\mathcal{O}\left(10^{-9}\right)[36-39]$ at $25 \mathrm{~Hz}$. Such a result constrains certain scenarios of PBHPBH mergers [9], cosmic string network [23] as well as the strong first-order phase transition at a very high scale $[28,29]$.

It has been demonstrated that a GW experiment can also be used to look for dark matter candidates, in both ultraheavy (see Ref. [40] and references therein) and ultralight [41-46] mass regions. So far, most of the PBH searches using SGWB are focused on PBHPBH mergers. However if PBHs exist, mergers between a PBH and an astrophysical objects naturally arises. For example, a PBH could merge with an ABH in stellar clusters and reproduce the LIGO/Virgo detection rate if the local overdensity of PBHs is large enough [47]. In this paper, we study the SGWB produced by mergers of a $\mathrm{PBH}$ and an ABH. All mergers in galaxies with different redshifts contribute to the isotropic SGWB. Meanwhile, coalescences that occur in the Milky Way (MW) can also generate a signal with a preferred direction. Both scenarios are considered in this study.

The paper is organized as follows. In Sec. 2, we provide the master formula and all the ingredients for estimating the isotropic SGWB. We compare our result with other sources of SGWB as well as the sensitivities of existing and future experiments. In Sec. 3, we estimate the $\mathrm{PBH}-\mathrm{ABH}$ formation rate in the MW and study whether the GW produced is proper to be treated as a contribution to the anisotropic SGWB. Finally, we conclude in Sec. 4.

\section{Isotropic Cosmological Background}

\subsection{GW power spectral density}

The isotropic SGWB is characterized by GW power spectral density $\Omega_{\mathrm{GW}}(\nu)$, which is a dimensionless quantity describing the GW energy density per logarithmic frequency interval,

$$
\Omega_{\mathrm{GW}}(\nu) \equiv \frac{1}{\rho_{c}} \frac{d \rho_{\mathrm{GW}}}{d \ln \nu}=\frac{\nu}{\rho_{c}} \frac{d \rho_{\mathrm{GW}}}{d \nu},
$$

where $\rho_{c}=3 H_{0}^{2} /(8 \pi G)$ is the critical energy density of the Universe. $H_{0}$ and $G$ are the Hubble constant and the gravitational constant. Here, $\rho_{\mathrm{GW}}$ is the energy density of the GW, and $\nu$ is the frequency of the GW observed today.

The GW power spectral density consists of GW radiation emitted throughout the entire history of the Universe. For the SGWB generated by ABH-PBH mergers, $\Omega_{\mathrm{GW}}$ can be written as an integral over the redshift:

$$
\Omega_{\mathrm{GW}}(\nu)=\frac{\nu}{\rho_{c} H_{0}} \int_{0}^{z_{\max }} d z \frac{R_{\mathrm{AP}}(z)}{(1+z)^{4} E(z)} \frac{d E_{\mathrm{GW}}}{d \nu_{s}}\left(\nu_{s}\right) .
$$

Here, $\frac{d E_{\mathrm{GW}}}{d \nu_{s}}\left(\nu_{s}\right)$ is the GW radiation energy spectrum of the source, $\nu_{s}$ is the GW frequency at the time of ABH-PBH merger, and it is related to the frequency at observation as $\nu_{s}=(1+z) \nu$. $R_{\mathrm{AP}}(z)$ is the $\mathrm{ABH}-\mathrm{PBH}$ merger rate, i.e., the number of mergers per physical volume per cosmological time. At last, $E(z)$ is related to the Hubble parameter as $E(z) \equiv H(z) / H_{0}$. 


\begin{tabular}{cccc}
\hline \hline Parameter & $a_{j}$ & $b_{j}$ & $c_{j}$ \\
\hline$\nu_{\text {merg }}$ & $2.9740 \times 10^{-1}$ & $4.4810 \times 10^{-2}$ & $9.5560 \times 10^{-2}$ \\
$\nu_{\text {ring }}$ & $5.9411 \times 10^{-1}$ & $8.9794 \times 10^{-2}$ & $1.9111 \times 10^{-1}$ \\
$\sigma$ & $5.0801 \times 10^{-1}$ & $7.7515 \times 10^{-2}$ & $2.2369 \times 10^{-2}$ \\
$\nu_{\text {cut }}$ & $8.4845 \times 10^{-1}$ & $1.2848 \times 10^{-1}$ & $2.7299 \times 10^{-1}$ \\
\hline \hline
\end{tabular}

Table 1: Parameters for the GW radiation power spectrum.

Therefore, the calculation of $\Omega_{\mathrm{GW}}(\nu)$ boils down to the GW radiation energy spectrum for each $\mathrm{ABH}-\mathrm{PBH}$ merger $\frac{d E_{\mathrm{GW}}}{d \nu_{s}}$ and the merger rate $R_{\mathrm{AP}}(z)$. We will present details on how they are calculated in the later sections. Notice that we impose an upper limit on redshift, $z_{\max }$, in the integral. This is because while PBHs can be formed formed at very large redshift deeply within the radiation-dominated epoch, ABHs can only appear after stars in galaxies have formed. Therefore $z_{\max }$ refers to the maximal redshift beyond which there is effectively no $\mathrm{ABH}$ and consequently no ABH-PBH merger.

\subsection{GW radiation power spectrum}

The evolution of a binary merger can be described by three phases: the inspiral, the merger and the ringdown. While the GW radiation from the early inspiral and ringdown phases can be approximated analytically by post-Newtonian expansion and perturbation theory, modeling the late inspiral and merger requires solving the Einstein equations numerically. Using the hybrid GW waveform for non-spinning binaries presented in Ref. [48], the GW energy spectrum can be written as

$$
\frac{d E_{\mathrm{GW}}}{d \nu_{s}}=\frac{(G \pi)^{2 / 3} M_{c}^{5 / 3}}{3} \times \begin{cases}\nu_{s}^{-1 / 3}, & \text { if } \nu_{s}<\nu_{\text {merg }} ; \\ \nu_{\mathrm{merg}}^{-1} \nu_{s}^{2 / 3}, & \text { if } \nu_{\text {merg }} \leq \nu_{s}<\nu_{\text {ring }} \\ \nu_{\text {merg }}^{-1} \nu_{\text {ring }}^{-4 / 3} \nu_{s}^{2}\left[\left(\frac{\nu_{s}-\nu_{\text {ring }}}{\sigma / 2}\right)^{2}+1\right]^{-2}, & \text { if } \nu_{\text {ring }} \leq \nu_{s}<\nu_{\text {cut }}\end{cases}
$$

in which $M_{c}=\left(m_{1} m_{2}\right)^{3 / 5} /\left(m_{1}+m_{2}\right)^{1 / 5}$ is the chirp mass of the binary. The frequencies $\nu_{\text {merg }}$ and $\nu_{\text {ring }}$ are boundaries that separate the contributions from different regimes - the inspiral, the merger and the ringdown stages. The parameter $\sigma$ characterizes the width of the transition from the merger stage to the ringdown stage, and $\nu_{\text {cut }}$ is the cutoff of this template. The frequency dependent behaviors of these parameters are summarized in a vector form $\vec{\alpha} \equiv\left\{\nu_{\text {merg }}, \nu_{\text {ring }}, \sigma, \nu_{\text {cut }}\right\}$ and can then be further parametrized as

$$
\alpha_{j}=\frac{a_{j} \eta^{2}+b_{j} \eta+c_{j}}{\pi} \times \frac{c^{3}}{M G}
$$

with $\eta=m_{1} m_{2} /\left(m_{1}+m_{2}\right)^{2}$ as the symmetric mass ratio, and $M$ as the total mass of the binary. The values of $\left(a_{j}, b_{j}, c_{j}\right)$ are listed in Table 1.

\section{$2.3 \quad$ Merger Rate}

ABHs follow the star distribution in galaxies. Thus the ABH-PBH merger rate can be calculated by integrating the number density of galaxies with the merger rate in each galaxy halo. 
To be specific, we have

$$
R_{\mathrm{AP}}(z)=\sum_{i} \int d M_{h} \frac{d n_{h}\left(z, M_{h}, i\right)}{d M_{h}} R_{\mathrm{AP}}^{\mathrm{halo}}\left(z, M_{h}, i\right),
$$

where $i$ indicates the type of a galaxy. It includes disk galaxies and elliptical galaxies in this study. $R_{\mathrm{AP}}^{\text {halo }}\left(z, M_{h}, i\right)$ is the merger rate of a type- $i$ halo with mass $M_{h}$ at redshift $z$, and $d n_{h} / d M_{h}$ is the halo mass function with $n_{h}$ being the physical number density of halos, which can be obtained through numerical simulations.

In order to estimate the merger rate $R_{\mathrm{AP}}^{\text {halo }}\left(z, M_{h}, i\right)$, the following ingredients are necessary: 1) the spatial distribution function of $\left.\mathrm{PBHs} n_{P}(\vec{r}) ; 2\right)$ the spatial distribution function of ABHs $n_{A}(\vec{r})$; 3) ABH-PBH binary formation probability, characterized by the averaged capture cross section $\left\langle\sigma_{\text {mer }} v_{\text {rel }}\right\rangle$. With these at hand, the ABH-PBH merger rate per halo can be written as ${ }^{1}$

$$
R_{\mathrm{AP}}^{\text {halo }}=\int_{\text {halo }} d V \int d M_{A} n_{P}\left(M_{h}, z, \vec{r}\right) \times \frac{d n_{A}}{d M_{A}}\left(M_{A}, M_{h}, z, \vec{r}\right) \times\left\langle\sigma_{\text {mer }}\left(M_{A}, M_{P}, v_{\text {rel }}\right) v_{\text {rel }}\right\rangle,
$$

where we keep the explicit dependence on the redshift $z$, the halo mass $M_{h}$, the $\mathrm{ABH} / \mathrm{PBH}$ masses, as well as the spatial location of the black holes $\vec{r}$ inside the halo. In the rest of this section, we provide details for these three ingredients and then combine them with the halo mass function to estimate the integrated merger rate.

\subsubsection{PBH distribution}

The mass of the $\mathrm{PBH}$ is not well predicted theoretically. In this study, we assume it takes a single value, and we consider two benchmarks, $M_{P}=1$ and $30 M_{\odot}$. Since the PBHs are produced at the very early time of the Universe, its spatial distribution follows that of dark matter. Assuming PBHs constitute a fraction, $f$, of the total dark matter abundance, the $\mathrm{PBH}$ number density can be written as

$$
n_{P}=\frac{\rho_{P}}{M_{P}}=f \times \frac{\rho_{\mathrm{dm}}}{M_{P}} .
$$

Here we assume the dark-matter distribution $\rho_{\mathrm{dm}}$ is described by the NFW profile [50],

$$
\rho_{\mathrm{dm}}(r)=\frac{\rho_{0}}{r / R_{s}\left(1+r / R_{s}\right)^{2}},
$$

where $r$ is the distance from the center of the halo, $\rho_{0}$ is the normalization factor and $R_{s}$ is related to the virial radius via the concentration parameter $C\left(M_{h}, z\right)=R_{\mathrm{vir}} / R_{s}$. In this study, we determine the concentration parameter using the fitting formula in Ref. [51] and only consider $C\left(M_{h}, z\right)$ between 0.5 and 1000 in order to avoid a divergent result [8].

The virial radius of a halo is defined through the averaged density within the region. More explicitly, for a halo at redshift $z$, one has

$$
M_{h} \simeq \Delta \times\left[\Omega_{m}(1+z)^{3}+\Omega_{\Lambda}\right] \rho_{c} \times \frac{4 \pi}{3} R_{\mathrm{vir}}^{3},
$$

where $\Delta$ is typically taken as $200[52]$.

\footnotetext{
${ }^{1}$ In this formula, the merger rate is identified with the binary formation rate. This is reasonable for the binary formation process that we study here in which the delay between the formation of the binary and the subsequent GW emission is negligible compared to cosmological timescales [49]. Such binary formation channel assumed here is consistent with the classical isolated single and binary evolution which follows the star formation rate.
} 


\subsubsection{ABH distribution}

The $\mathrm{ABH}$ mass ranges from $\sim 5 M_{\odot}$ to a few tens of solar masses $[53,54]$. In order to calculate the distribution of $\mathrm{ABHs}$ with respect to different masses, we use the initial mass function (IMF) [55] which describes the number distribution of stars:

$$
\frac{d N_{M}}{d M} \propto\left\{\begin{array}{ll}
M^{-0.3}, & 0.01 M_{\odot} \leq M<0.08 M_{\odot} \\
M^{-1.3}, & 0.08 M_{\odot} \leq M<0.5 M_{\odot} \\
M^{-2.3}, & 0.5 M_{\odot} \leq M<100 M_{\odot}
\end{array} .\right.
$$

To determine the fraction of stars that eventually forms black holes, we make a simple assumption that only stars with masses larger than $25 M_{\odot}$ become black holes. ${ }^{2}$ Moreover, although the formation of a black hole takes a finite amount of time, the lifetime of a star with its mass larger than $25 M_{\odot}$ is quite small compared with the cosmological time that we consider in this paper. Therefore, we ignore the $\mathrm{ABH}$ formation time and estimate its number density per solar mass of stellar objects as

$$
\frac{\chi_{A}\left(M_{A}\right)}{M_{\odot}} \simeq \frac{\left.\frac{d N_{M}}{d M}\right|_{M=M_{A}^{\text {pro }}}}{\int_{0.08 M_{\odot}}^{100 M_{\odot}} M \frac{d N_{M}}{d M} d M} .
$$

For simplicity, we follow the similar approach in [64] and assume the remnant ABH only retains $\sim 1 / 3$ of the initial stellar mass. Therefore, $M_{A}^{\text {pro }} \simeq 3 M_{A}$ stands for the mass of the progenitor associated with an $\mathrm{ABH}$ of mass $M_{A}$. In reality, the remnant mass can be affected by stellar winds which depend on metallicity and thus also depend on redshift [65-68]. Integrating the equation above, we find that the averaged number of ABHs per solar mass is $\sim 2 \times 10^{-3}$. For a galaxy with mass distribution $\rho_{G}$, we thus have the spatial distribution of the $\mathrm{ABH}$ number density as a function of the $\mathrm{ABH}$ mass as

$$
\frac{d n_{A}}{d M_{A}}=\frac{\chi_{A}\left(M_{A}\right)}{M_{\odot}} \times \rho_{G}
$$

Notice that this relation assumes that all stars and thus all ABHs are formed in isolation and in the field. In practice, the majority of them are born in binary systems [69, 70], which might further affect our assumption of two-body capture. We shall reserve the consideration of such effect for future work.

The distribution $\rho_{G}$ depends on multiple aspects of a galaxy, i.e., the redshift, the halo mass and also the type of the galaxy. For elliptical galaxies, we take the Hernquist Model [71]

$$
\rho_{G}(r)=\frac{C_{E}}{2 \pi} \frac{R_{c}}{r\left(r+R_{c}\right)^{3}},
$$

in which $r$ is the radius in spherical coordinates, and the core radius $R_{c}$ can be determined by its relation to the half-light (half-mass) radius $(\sqrt{2}+1) R_{c}=R_{1 / 2}$. For disk galaxies, the

\footnotetext{
${ }^{2}$ This assumption follows from the conclusion in Ref. [56] that a star with metallicity between metal-free and solar metallicity becomes a black hole by supernova mass fallback or direct core collapse if its mass is larger than $25 M_{\odot}$, and those with lower masses can only form white dwarfs or neutron stars. However, recent studies show that the relation between neutron star and black-hole formation is more sophisticated and there is not a single critical mass above which black holes can form [57-63].
} 


\begin{tabular}{cccccccc}
\hline \hline$M_{0}$ & $M_{a}$ & $M_{\ln a}$ & $M_{z}$ & $\epsilon_{0}$ & $\epsilon_{a}$ & $\epsilon_{\ln a}$ & $\epsilon_{z}$ \\
12.06 & 4.609 & 4.525 & -0.756 & -1.459 & 1.515 & 1.249 & -0.214 \\
\hline$\alpha_{0}$ & $\alpha_{a}$ & $\alpha_{\ln a}$ & $\alpha_{z}$ & $\beta_{0}$ & $\beta_{a}$ & - & $\beta_{z}$ \\
1.972 & -2.523 & -1.868 & 0.188 & 0.488 & -0.965 & - & -0.569 \\
\hline$\gamma_{0}$ & $\gamma_{a}$ & - & $\gamma_{z}$ & $\delta_{0}$ & - & - & - \\
-0.958 & -2.230 & - & -0.706 & 0.391 & - & - & - \\
\hline \hline
\end{tabular}

Table 2: Parameters for the stellar-halo mass relation.

mass distribution is approximately described by a double exponential form [72]:

$$
\rho_{G}(R, h)=C_{D} \exp \left(-R / R_{D}\right) \exp \left(-|h| / h_{D}\right),
$$

where $R$ and $h$ are the radius and height in cylindrical coordinates. $R_{D}$ and $h_{D}$ are related to the half-light radius of the halo hosting the galaxy as $1.68 R_{D} \simeq R_{1 / 2}$, and $h_{D} \simeq R_{D} / 10$.

In both cases, we have $R_{1 / 2}=\lambda R_{\text {vir }}$ with $\lambda \simeq 0.015$ [73]. Moreover, the normalization factor $C_{D, E}$ in both profiles are determined by

$$
\int_{\text {halo }} d V \rho_{G}=M_{s}
$$

where $M_{s}$ is the total stellar mass in the galaxy.

We determine the total stellar mass using the stellar-halo mass relation provided in Ref. [74]. In particular, the stellar-halo mass relations are parametrized as

$$
\log _{10}\left(\frac{M_{s}}{M_{1}}\right)=\epsilon-\log _{10}\left(10^{-\alpha x}+10^{-\beta x}\right)+\gamma \exp \left[-\frac{1}{2}\left(\frac{x}{\delta}\right)^{2}\right],
$$

in which $x=\log _{10}\left(M_{h} / M_{1}\right)$. The parameters scales with redshift as

$$
\begin{aligned}
\log _{10}\left(\frac{M_{1}}{M_{\odot}}\right) & =M_{0}+M_{a}(a-1)-M_{\ln a} \ln a+M_{z} z, \\
\epsilon & =\epsilon_{0}+\epsilon_{a}(a-1)-\epsilon_{\ln a} \ln a+\epsilon_{z} z, \\
\alpha & =\alpha_{0}+\alpha_{a}(a-1)-\alpha_{\ln a} \ln a+\alpha_{z} z, \\
\beta & =\beta_{0}+\beta_{a}(a-1)+\beta_{z} z \\
\log _{10} \gamma & =\gamma_{0}+\gamma_{a}(a-1)+\gamma_{z} z \\
\delta & =\delta_{0}
\end{aligned}
$$

in which $a \equiv 1 /(1+z)$ is the scale factor, and the values of the additional parameters are summarized in Table 2. A few examples of the stellar-halo mass relation at different redshifts are shown in FIG. 1.

In our analysis, we consider two limits where all galaxies in the Universe are either disk galaxies or elliptical galaxies. The reality should lie between the results from these two limits.

\subsubsection{ABH-PBH binary formation probability}

We estimate the ABH-PBH binary merger rate through gravitational capture process. As an $\mathrm{ABH}$ and a $\mathrm{PBH}$ pass each other, the gravitational wave radiation takes away some amount of the energy of the system. If the energy loss is large enough and brings the total energy of the 


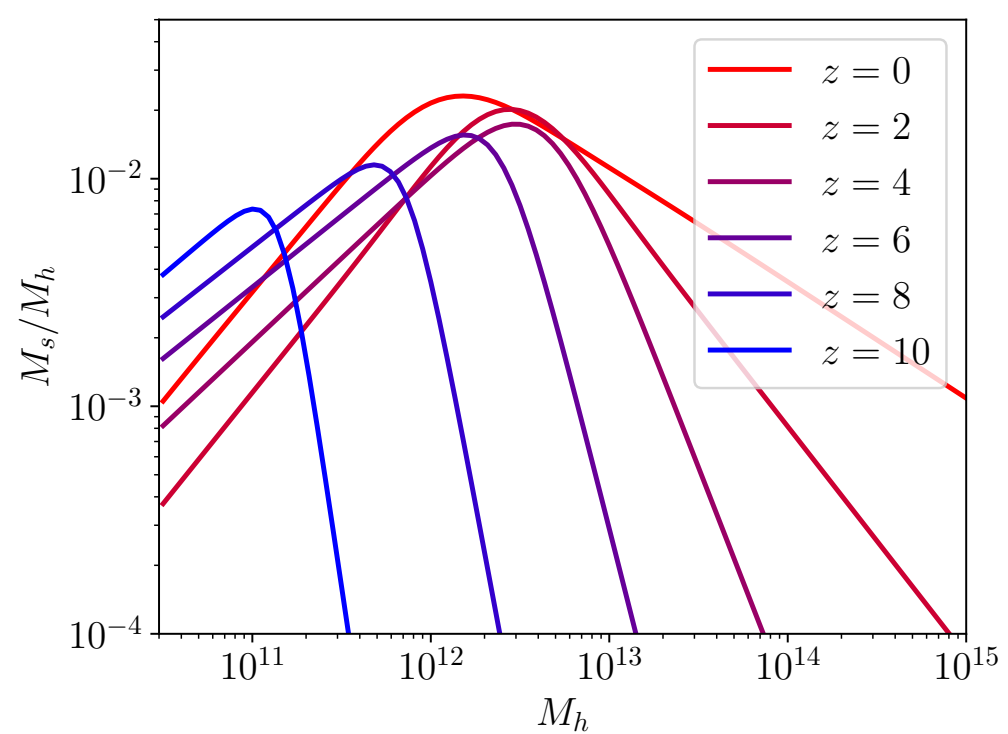

FIG. 1. Stellar-halo mass relation.

system below zero, a bound state forms, and the merger will happen soon after. For binaries formed via this mechanism, the characteristic delay time, describing the duration between the binary formation and the final coalescence, depends on the velocity dispersion of the hosting halo. Is typically much shorter (hours for $M_{h} \sim 10^{12} M_{\odot}$ and kyrs for $M_{h} \sim 10^{6} M_{\odot}$ [49]) compared to the Hubble time. Therefore we can safely treat it as instantaneous on cosmological time scales. The gravitational capture cross-section can be estimated as [75]

$$
\sigma_{\mathrm{mer}}\left(m_{i}, m_{j}\right)=2 \pi\left(\frac{85 \pi}{6 \sqrt{2}}\right)^{2 / 7} \frac{G^{2}\left(m_{i}+m_{j}\right)^{10 / 7} m_{i}^{2 / 7} m_{j}^{2 / 7}}{c^{10 / 7} v_{\mathrm{rel}}^{18 / 7}}
$$

where $m_{i}$ and $m_{j}$ are the masses of two black holes which will be identified as the ABH mass $M_{A}$ and the $\mathrm{PBH}$ mass $M_{P}$ respectively, and $v_{\text {rel }}$ is the relative velocity between these two black holes.

We assume both the ABH and the PBH velocities follow the same Maxwell-Boltzmann distribution with a cutoff at the virial velocity, $v_{\text {vir }} \equiv \sqrt{2 G M_{h} / R_{\text {vir }}}$, of a halo with mass $M_{h}$ $[8,49]$ :

$$
\begin{aligned}
P\left(v, v_{\mathrm{m}}\right) & =F_{0}\left[\exp \left(-\frac{v^{2}}{v_{\mathrm{m}}^{2}}\right)-\exp \left(-\frac{v_{\mathrm{vir}}^{2}}{v_{\mathrm{m}}^{2}}\right)\right], \\
v_{\mathrm{m}} & =\frac{v_{\text {vir }}}{\sqrt{2}} \sqrt{\frac{C}{C_{m}} \frac{g\left(C_{m}\right)}{g(C)}}, \\
g(X) & =\ln (1+X)-\frac{1}{1+X},
\end{aligned}
$$

where $v_{\mathrm{m}}$ is the maximum circular velocity in an NFW halo which occurs at $R_{m}=C_{m} R_{s}$ with $C_{m}=2.1626$, and $F_{0}$ is the normalization factor so that $\int_{0}^{v_{\mathrm{vir}}} d v 4 \pi v^{2} P\left(v, v_{\mathrm{m}}\right)=1$. 


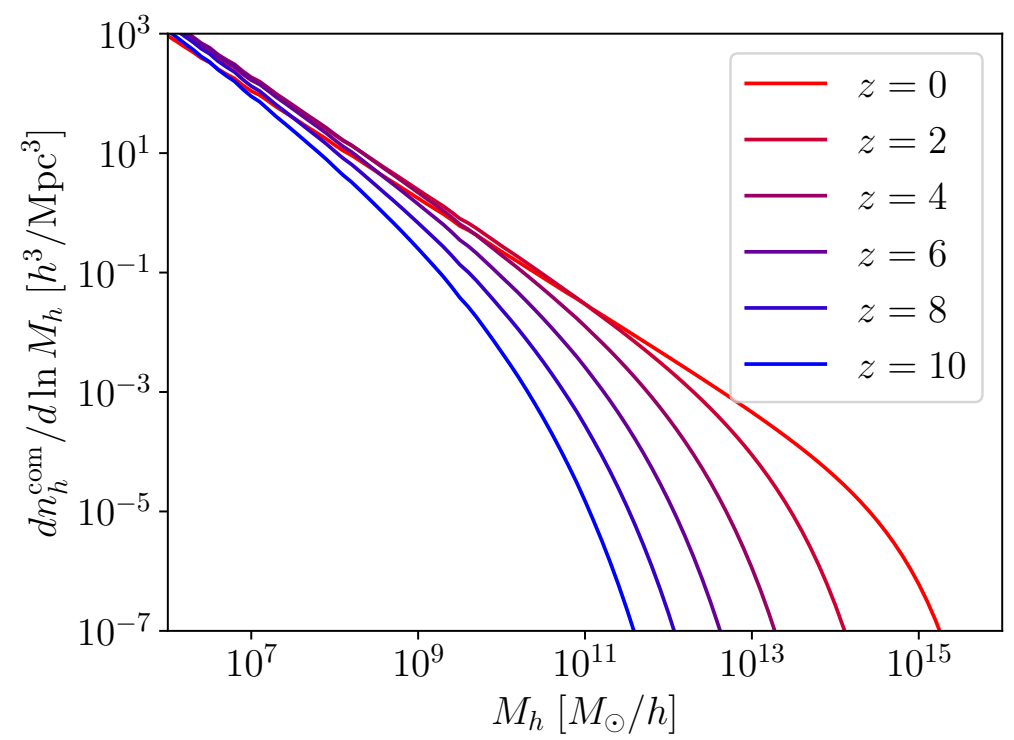

FIG. 2. The Sheth-Tormen halo mass function in the comoving frame.

Therefore, averaged cross-section in a halo is defined as

$$
\left\langle\sigma_{\mathrm{mer}} v\right\rangle \equiv \int d^{3} v_{1} d^{3} v_{2} \sigma_{\mathrm{mer}} v_{\mathrm{rel}} P\left(v_{1}, v_{m}\right) P\left(v_{2}, v_{m}\right),
$$

in which $v_{1}$ and $v_{2}$ are the velocities of ABHs and PBHs respectively, and $v_{\text {rel }}=\left|\vec{v}_{1}-\vec{v}_{2}\right|$.

\subsubsection{Halo mass function}

For the halo mass distribution, we adopt the Sheth-Tormen halo mass function [76] which is an extension to the Press-Schechter formalism [77] that fits well with the results of numerical simulations. Examples of the halo mass function at several different redshifts are provided in FIG. 2. Notice that the examples are shown in the comoving frame, rather than the physical volume.

\subsection{Isotropic SGWB power spectral density from ABH-PBH merger}

With all the ingredients prepared, the SGWB can be estimated by inserting Eq. (2.3) and Eq. (2.5) into Eq. (2.2). The SGWB energy density spectra are shown in Fig. 3. Here the two benchmark values of the primordial black hole mass, $1 M_{\odot}$ and $30 M_{\odot}$, are presented by assuming $f=1$. Since the merger rate depends the PBH fraction $f$ linearly, results for different $f$ can be easily inferred. Due to the large astrophysical uncertainties on the star population at high redshift, we present results with different choices of the cutoff redshift as $z_{\max }=5$ and 10 .

Notice that choosing different $z_{\max }$ has a noticeable effect. Obviously, a larger $z_{\max }$ means more contribution from higher redshift which enhances the spectrum at lower frequency. Consequently, we observe that the peak shifts to a lower frequency. At a larger redshift, the validity of our astrophysical inputs may not be applicable. Therefore, we do not extend our calculation to a redshift higher than $z=10$. 

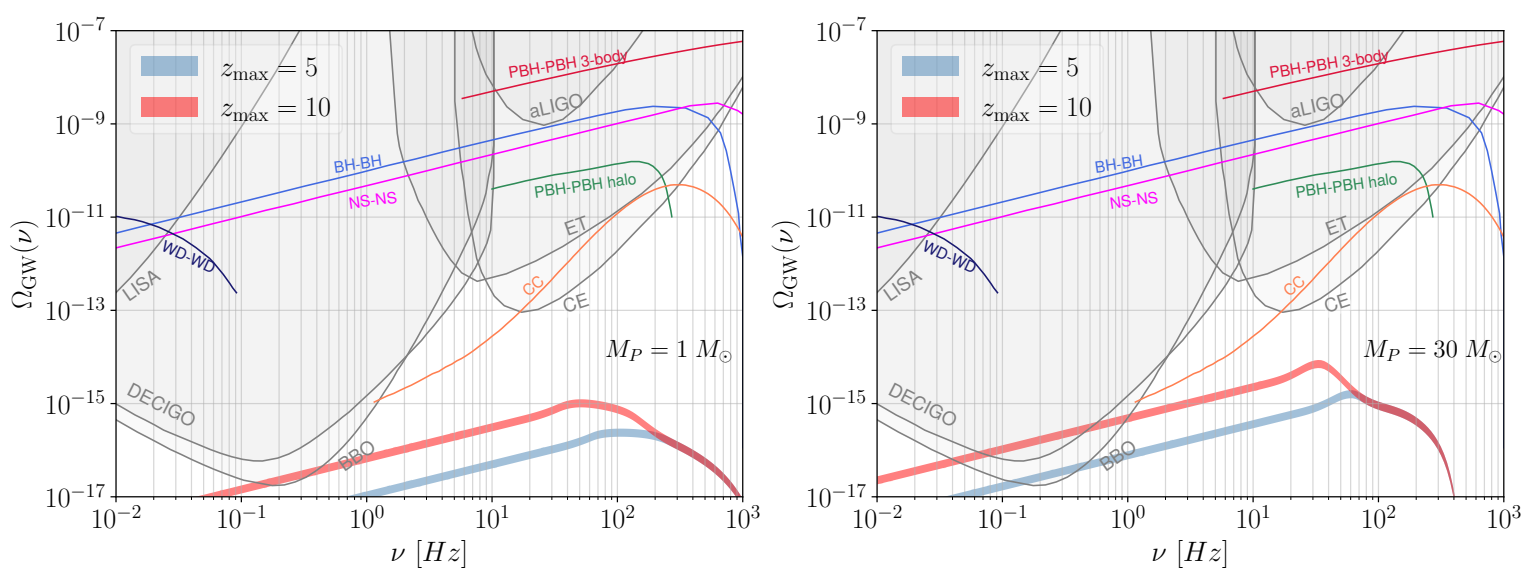

FIG. 3. Here we show the isotropic component of the stochastic GW background from ABH-PBH mergers. The left and right panels are results for primordial black hole mass as 1 and $30 M_{\odot}$, respectively. For each colored band, the upper boundary is obtained by assuming $100 \%$ elliptical galaxies, whereas the lower boundary assumes $100 \%$ disk galaxies. Various colored bands correspond to different choices of redshift cutoff, $z_{\max }$. The sensitivities for several existing and future GW experiments [78] are shown as the gray curves. We also present the expected SGWB produced by core collapse (CC) [79] and other types of binaries, including PBH-PBH binaries formed at early time through 3-body process [9] (with chirp mass as $1 M_{\odot}, f=0.05$ ), PBH-PBH binaries through gravitational capture in dark matter halos [8] (with chirp mass as $30 M_{\odot}, f=1$ ), WD-WD binaries [80], as well as ABH-ABH and NS-NS binaries [36]. Note that we simply take representative results from these references and all the expected signals are subject to large uncertainties.

Another noticeable difference for the two choices of $M_{P}$ is the overall magnitude of $\Omega_{\mathrm{GW}}$ - the one with larger $M_{P}$ has a larger GW density spectrum. This can be understood as follows. The peak of $d E_{\mathrm{GW}} / d \ln \nu_{s}$ scales as $M_{c}^{5 / 3} \nu_{\text {merg }}^{-1} \nu_{\text {ring }}^{2 / 3} \sim M_{A} M_{P}$. At the same time, the capture rate is proportional to $n_{P} \sigma_{\text {mer }}$ which scales as $M_{P}^{-1}\left(M_{A}+M_{P}\right)^{10 / 7} M_{A}^{2 / 7} M_{P}^{2 / 7}$. Clearly, a larger $M_{P}$ gives rise to a higher GW spectrum.

For each colored band in the plot, the upper boundary is obtained by assuming all elliptical galaxies, while the lower boundary corresponds to the assumption of $100 \%$ disk galaxies. The width of the band characterizes the uncertainty from the galaxy type.

The peak of the SGWB energy density from ABH-PBH mergers falls between $\mathcal{O}\left(10^{-15}\right.$ $\left.10^{-14}\right)$. This is far below the sensitivities of the existing or future ground-based experiments such as the aLIGO, Einstein Telescope (ET), Cosmic Explorer (CE). For future space-based experiments, the Deci-Hertz Interferometer Gravitational-Wave Observatory (DECIGO) and the Big-Bang Observer (BBO) may have sensitivities to probe the ABH-PBH SGWB at a lower frequency band. However distinguishing such a spectrum from the astrophysical background remains challenging.

\section{PBH-ABH binaries in the Milky Way}

The previous discussion is based on the isotropic distribution of galaxies in our Universe. The ABH-PBH mergers within our MW may also contribute to an anisotropic SGWB. These mergers are very close to us and we should be able to identify them one-by-one. However, 


\begin{tabular}{ccc}
\hline \hline$\rho_{b, 0}$ & $r_{0}$ & $r_{\text {cut }}$ \\
$95.6 M_{\odot} \mathrm{pc}^{-3}$ & $0.075 \mathrm{kpc}$ & $2.1 \mathrm{kpc}$ \\
\hline$\Sigma_{d, 0, \text { thin }}$ & $h_{d, \text { thin }}$ & $R_{d, \text { thin }}$ \\
$816.6 M_{\odot} \mathrm{pc}^{-2}$ & $0.3 \mathrm{kpc}$ & $2.9 \mathrm{kpc}$ \\
\hline$\Sigma_{d, 0, \text { thick }}$ & $h_{d, \text { thick }}$ & $R_{d, \text { thick }}$ \\
$209.5 M_{\odot} \mathrm{pc}^{-2}$ & $0.9 \mathrm{kpc}$ & $3.31 \mathrm{kpc}$ \\
\hline \hline
\end{tabular}

Table 3: Parameters for MW components.

if we focus on the frequency regime which is much lower than that of LIGO, it may still be useful to consider the SGWB produced during the inspiral stage. In this section, we estimate the binary formation rate in the MW and see whether it is proper to be treated as a source of SGWB.

For $\rho_{\mathrm{MW}}$, we use the best-fitting mass model of the MW as well as its host halo provided in Ref. [81]. In this model, the MW consists of three components - the bulge, the thin disk and the thick disk. The bulge and the disk density profiles take the following form respectively

$$
\begin{aligned}
\rho_{b} & =\frac{\rho_{b, 0}}{\left(1+r^{\prime} / r_{0}\right)^{\alpha}} e^{-\left(r^{\prime} / r_{\mathrm{cut}}\right)^{2}}, \\
\rho_{d} & =\frac{\Sigma_{d, 0}}{2 h_{d}} e^{-\frac{|h|}{h_{d}}-\frac{R}{R_{d}}},
\end{aligned}
$$

in which $\alpha=1.8, R$ and $h$ are the radius and the height in cylindrical coordinates, and $r^{\prime}=\sqrt{R^{2}+(h / q)^{2}}$ with the axis ratio $q=0.5$. The dimensionful parameters are listed in Table 3 .

For the host halo, we still take the NFW profile in Eq. (2.8) with $\rho_{0}=0.00846 M_{\odot} / \mathrm{pc}^{3}$ and $R_{s}=20.2 \mathrm{kpc}$. We assume that the location of the solar system is right on the galactic disk $(h \simeq 0)$ at a distance $R_{\odot} \simeq 8.29 \mathrm{kpc}$ away from the galactic center.

With these profiles, we can then estimate the binary formation rate in the MW. Straightforward calculation shows that this rate is $\sim 2.29 \times 10^{-12} \mathrm{yr}^{-1}$ for $M_{P}=1 M_{\odot}$ and $\sim 8.32 \times 10^{-13} \mathrm{yr}^{-1}$ for $M_{P}=30 M_{\odot}$. The binary formation rate is so low that it is not likely to have even a single merger event during the age of the Universe. Therefore, it is not appropriate to treat the ABH-PBH mergers in the MW as a source of SGWB.

\section{Conclusion}

In this paper, we study the SGWB produced by unresolved PBH-ABH mergers. We demonstrate that, in the higher frequency region, i.e., $\mathcal{O}(10-1000) \mathrm{Hz}$, the GW radiation is much lower than the reach of any existing or future ground-based GW experiment. In the lower frequency region, it may be within the reach of future space-based experiments such as DECIGO and BBO. Thus the SGWB produced by $\mathrm{PBH}-\mathrm{ABH}$ mergers is not the key component leading to the discovery of PBHs. The uncertainty due to the type of galaxies (disk or elliptical) is relatively small. On the other hand, the uncertainty due to the choice of the cutoff redshift has a noticeable effect in both the magnitude and the shape of the power spectrum. In this paper, we assumed that all PBHs have the same mass. In more realistic PBH models, the PBHs may have a broader mass spectrum. Moreover, the duration between the bound state formation and the merger of the binary is neglected. This is a safe approximation because 
it is much shorter than the time scale we are interested in [49]. In addition, the PBHs are assumed to have a spatial distribution that follows the NFW profile. A change in the spatial distribution, such as the clustering of PBHs, might help increase the merger rate [82].

The SGWB signal from PBH-ABH mergers are subject to large background. For example, it is several orders of magnitude below the estimated backgrounds from WD-WD, NS-NS, and $\mathrm{ABH}-\mathrm{ABH}$ mergers. In practice, it is very challenging to detect the SGWB signal from the PBH-ABH mergers.

Furthermore, the SGWB from PBH-ABH mergers also naturally constitute an inevitable background for PBH-PBH mergers. Since the estimated PBH-ABH signal is much smaller than the PBH-PBH signal, our results illustrate that previous analysis for $\mathrm{PBH}-\mathrm{PBH}$ mergers $[8,9]$ are still valid and not affected by this natural background.

\section{Acknowledgments}

We would like to thank Huanian Zhang and Zheng Zheng for useful discussions. J.S. and F.H. are supported by the National Natural Science Foundation of China under Grants No. 12025507, 11690022, 11947302; and by the Strategic Priority Research Program and Key Research Program of Frontier Science of the Chinese Academy of Sciences under Grants No. XDB21010200, XDB23010000, ZDBS-LY-7003, and also by the CAS Project for Young Scientists in Basic Research under Grant No. YSBR-006. F.H. is also supported by the National Science Foundation of China under Grants No. 12022514 and No. 11875003. Y.Z. is supported by U.S. Department of Energy under Award No. DESC0009959. Y.Z. would like to thank the ITP-CAS for their kind hospitality.

\section{References}

[1] LIGO Scientific, Virgo collaboration, Observation of Gravitational Waves from a Binary Black Hole Merger, Phys. Rev. Lett. 116 (2016) 061102 [1602.03837].

[2] J. Aasi, B. Abbott, R. Abbott, T. Abbott, M. Abernathy, K. Ackley et al., Advanced ligo, Classical and quantum gravity 32 (2015) 074001.

[3] F. a. Acernese, M. Agathos, K. Agatsuma, D. Aisa, N. Allemandou, A. Allocca et al., Advanced virgo: a second-generation interferometric gravitational wave detector, Classical and Quantum Gravity 32 (2014) 024001.

[4] B. Abbott, R. Abbott, T. Abbott, S. Abraham, F. Acernese, K. Ackley et al., Gwtc-1: a gravitational-wave transient catalog of compact binary mergers observed by ligo and virgo during the first and second observing runs, Physical Review X 9 (2019) 031040.

[5] R. Abbott, T. Abbott, S. Abraham, F. Acernese, K. Ackley, A. Adams et al., Gwtc-2: Compact binary coalescences observed by ligo and virgo during the first half of the third observing run, arXiv preprint arXiv:2010.14527 (2020) .

[6] LIGO ScIEntific, KAGRA, VIRGO collaboration, Observation of Gravitational Waves from Two Neutron Star-Black Hole Coalescences, Astrophys. J. Lett. 915 (2021) L5 [2106.15163].

[7] LIGO ScIENTIFIC, VIRGO collaboration, GW150914: Implications for the stochastic gravitational wave background from binary black holes, Phys. Rev. Lett. 116 (2016) 131102 [1602.03847].

[8] V. Mandic, S. Bird and I. Cholis, Stochastic Gravitational-Wave Background due to Primordial Binary Black Hole Mergers, Phys. Rev. Lett. 117 (2016) 201102 [1608.06699]. 
[9] S. Wang, Y.-F. Wang, Q.-G. Huang and T. G. F. Li, Constraints on the Primordial Black Hole Abundance from the First Advanced LIGO Observation Run Using the Stochastic Gravitational-Wave Background, Phys. Rev. Lett. 120 (2018) 191102 [1610.08725].

[10] Z.-C. Chen, F. Huang and Q.-G. Huang, Stochastic Gravitational-wave Background from Binary Black Holes and Binary Neutron Stars and Implications for LISA, Astrophys. J. 871 (2019) 97 [1809.10360].

[11] S. Mukherjee and J. Silk, Can we distinguish astrophysical from primordial black holes via the stochastic gravitational wave background?, 2105.11139.

[12] S. Mukherjee, M. S. P. Meinema and J. Silk, Prospects of discovering sub-solar primordial black holes using the stochastic gravitational wave background from third-generation detectors, 2107.02181.

[13] S. S. Bavera, G. Franciolini, G. Cusin, A. Riotto, M. Zevin and T. Fragos, Stochastic gravitational-wave background as a tool to investigate multi-channel astrophysical and primordial black-hole mergers, 2109.05836.

[14] S. Marassi, R. Schneider and V. Ferrari, Gravitational wave backgrounds and the cosmic transition from Population III to Population II stars, Mon. Not. Roy. Astron. Soc. 398 (2009) 293 [0906. 0461].

[15] X.-J. Zhu, E. Howell and D. Blair, Observational upper limits on the gravitational wave production of core collapse supernovae, Mon. Not. Roy. Astron. Soc. 409 (2010) L132 [1008.0472].

[16] A. Buonanno, G. Sigl, G. G. Raffelt, H.-T. Janka and E. Muller, Stochastic gravitational wave background from cosmological supernovae, Phys. Rev. D 72 (2005) 084001 [astro-ph/0412277].

[17] P. Sandick, K. A. Olive, F. Daigne and E. Vangioni, Gravitational Waves from the First Stars, Phys. Rev. D 73 (2006) 104024 [astro-ph/0603544].

[18] T. Regimbau and V. Mandic, Astrophysical Sources of Stochastic Gravitational-Wave Background, Class. Quant. Grav. 25 (2008) 184018 [0806. 2794].

[19] S. Marassi, R. Ciolfi, R. Schneider, L. Stella and V. Ferrari, Stochastic background of gravitational waves emitted by magnetars, Mon. Not. Roy. Astron. Soc. 411 (2011) 2549 [1009.1240].

[20] Q. Cheng, Y.-W. Yu and X.-P. Zheng, Stochastic gravitational wave background from magnetic deformation of newly born magnetars, Mon. Not. Roy. Astron. Soc. 454 (2015) 2299 [1509.07651].

[21] Q. Cheng, S.-N. Zhang and X.-P. Zheng, Stochastic gravitational wave background from newly born massive magnetars: The role of a dense matter equation of state, Phys. Rev. D 95 (2017) 083003 [1704.02013].

[22] S. R. Chowdhury and M. Khlopov, The Stochastic Gravitational Wave Background from Magnetars, Universe 7 (2021) 381 [2110.07655].

[23] LigO Scientific, Virgo, KAGRA collaboration, Constraints on Cosmic Strings Using Data from the Third Advanced LIGO-Virgo Observing Run, Phys. Rev. Lett. 126 (2021) 241102 [2101.12248].

[24] L. P. Grishchuk, Amplification of gravitational waves in an istropic universe, Zh. Eksp. Teor. Fiz. 67 (1974) 825.

[25] A. A. Starobinsky, Spectrum of relict gravitational radiation and the early state of the universe, JETP Lett. 30 (1979) 682.

[26] L. A. Boyle and A. Buonanno, Relating gravitational wave constraints from primordial 
nucleosynthesis, pulsar timing, laser interferometers, and the CMB: Implications for the early Universe, Phys. Rev. D 78 (2008) 043531 [0708.2279].

[27] LIGO ScIENTIFIC, VIRGO collaboration, Searching for stochastic gravitational waves using data from the two colocated LIGO Hanford detectors, Phys. Rev. D 91 (2015) 022003 [1410.6211].

[28] A. Romero, K. Martinovic, T. A. Callister, H.-K. Guo, M. Martínez, M. Sakellariadou et al., Implications for First-Order Cosmological Phase Transitions from the Third LIGO-Virgo Observing Run, Phys. Rev. Lett. 126 (2021) 151301 [2102.01714].

[29] F. Huang, V. Sanz, J. Shu and X. Xue, LIGO as a probe of Dark Sectors, 2102.03155.

[30] S. Vitale, Space-borne Gravitational Wave Observatories, Gen. Rel. Grav. 46 (2014) 1730 [1404.3136].

[31] LISA collaboration, Laser Interferometer Space Antenna, 1702.00786.

[32] W.-R. Hu and Y.-L. Wu, The Taiji Program in Space for gravitational wave physics and the nature of gravity, Natl. Sci. Rev. 4 (2017) 685.

[33] TianQin collaboration, TianQin: a space-borne gravitational wave detector, Class. Quant. Grav. 33 (2016) 035010 [1512.02076].

[34] R. Smits, M. Kramer, B. Stappers, D. R. Lorimer, J. Cordes and A. Faulkner, Pulsar searches and timing with the square kilometre array, Astron. Astrophys. 493 (2009) 1161 [0811.0211].

[35] G. Hobbs et al., The international pulsar timing array project: using pulsars as a gravitational wave detector, Class. Quant. Grav. 27 (2010) 084013 [0911.5206].

[36] LIGO ScIENTIFIC, VIRGO collaboration, Upper Limits on the Stochastic Gravitational-Wave Background from Advanced LIGO's First Observing Run, Phys. Rev. Lett. 118 (2017) 121101 [1612.02029].

[37] LIGO SCIENTIFIC, VIRGO collaboration, Search for the isotropic stochastic background using data from Advanced LIGO's second observing run, Phys. Rev. D 100 (2019) 061101 [1903.02886].

[38] LIGO ScIEnTIFIC, Virgo, KAGRA collaboration, Upper Limits on the Isotropic Gravitational-Wave Background from Advanced LIGO's and Advanced Virgo's Third Observing Run, 2101.12130.

[39] LIGO SCIEnTIfIC, VIRGO, KAGRA collaboration, The population of merging compact binaries inferred using gravitational waves through GWTC-3, 2111.03634.

[40] S. Clesse et al., Snowmass2021 - Letter of interest: Gravitational waves from primordial black holes, https:

//www. snowmass21. org/docs/files/summaries/CF/SNOWMASS21-CF3_CF7-091. pdf .

[41] A. Pierce, K. Riles and Y. Zhao, Searching for Dark Photon Dark Matter with Gravitational Wave Detectors, Phys. Rev. Lett. 121 (2018) 061102 [1801.10161].

[42] H.-K. Guo, K. Riles, F.-W. Yang and Y. Zhao, Searching for Dark Photon Dark Matter in LIGO O1 Data, Commun. Phys. 2 (2019) 155 [1905.04316].

[43] LiGO Scientific, Virgo, KAGRA collaboration, Constraints on dark photon dark matter using data from LIGO's and Virgo's third observing run, 2105.13085.

[44] A. L. Miller et al., Probing new light gauge bosons with gravitational-wave interferometers using an adapted semicoherent method, Phys. Rev. D 103 (2021) 103002 [2010.01925].

[45] H. Grote and Y. V. Stadnik, Novel signatures of dark matter in laser-interferometric gravitational-wave detectors, Phys. Rev. Res. 1 (2019) 033187 [1906.06193]. 
[46] S. M. Vermeulen et al., Direct limits for scalar field dark matter from a gravitational-wave detector, 2103.03783.

[47] K. Kritos, V. De Luca, G. Franciolini, A. Kehagias and A. Riotto, The Astro-Primordial Black Hole Merger Rates: a Reappraisal, JCAP 05 (2021) 039 [2012.03585].

[48] P. Ajith et al., A Template bank for gravitational waveforms from coalescing binary black holes. I. Non-spinning binaries, Phys. Rev. D77 (2008) 104017 [0710.2335].

[49] S. Bird, I. Cholis, J. B. Muñoz, Y. Ali-Haïmoud, M. Kamionkowski, E. D. Kovetz et al., Did LIGO detect dark matter?, Phys. Rev. Lett. 116 (2016) 201301 [1603.00464].

[50] J. F. Navarro, C. S. Frenk and S. D. White, A Universal density profile from hierarchical clustering, Astrophys. J. 490 (1997) 493 [astro-ph/9611107].

[51] F. Prada, A. A. Klypin, A. J. Cuesta, J. E. Betancort-Rijo and J. Primack, Halo concentrations in the standard LCDM cosmology, Mon. Not. Roy. Astron. Soc. 423 (2012) 3018 [1104.5130].

[52] M. J. White, The Mass of a halo, Astron. Astrophys. 367 (2001) 27 [astro-ph/0011495].

[53] C. D. Bailyn, R. K. Jain, P. Coppi and J. A. Orosz, The Mass distribution of stellar black holes, Astrophys. J. 499 (1998) 367 [astro-ph/9708032].

[54] W. M. Farr, N. Sravan, A. Cantrell, L. Kreidberg, C. D. Bailyn, I. Mandel et al., The Mass Distribution of Stellar-Mass Black Holes, Astrophys. J. 741 (2011) 103 [1011.1459].

[55] P. Kroupa, On the variation of the initial mass function, Mon. Not. Roy. Astron. Soc. 322 (2001) 231 [astro-ph/0009005].

[56] A. Heger, C. L. Fryer, S. E. Woosley, N. Langer and D. H. Hartmann, How massive single stars end their life, Astrophys. J. 591 (2003) 288 [astro-ph/0212469].

[57] E. O'Connor and C. D. Ott, Black Hole Formation in Failing Core-Collapse Supernovae, Astrophys. J. 730 (2011) 70 [1010.5550].

[58] M. Ugliano, H. T. Janka, A. Marek and A. Arcones, Progenitor-Explosion Connection and Remnant Birth Masses for Neutrino-Driven Supernovae of Iron-Core Progenitors, Astrophys. J. 757 (2012) 69 [1602.06327].

[59] O. Pejcha and T. A. Thompson, The Landscape of the Neutrino Mechanism of Core-Collapse Supernovae: Neutron Star and Black Hole Mass Functions, Explosion Energies and Nickel Yields, Astrophys. J. 801 (2015) 90 [1409.0540].

[60] T. Sukhbold, T. Ertl, S. E. Woosley, J. M. Brown and H. T. Janka, Core-Collapse Supernovae from 9 to 120 Solar Masses Based on Neutrino-powered Explosions, Astrophys. J. 821 (2016) 38 [1510.04643].

[61] T. Ertl, S. E. Woosley, T. Sukhbold and H. T. Janka, The Explosion of Helium Stars Evolved With Mass Loss, 1910.01641.

[62] R. A. Patton and T. Sukhbold, Towards a Realistic Explosion Landscape for Binary Population Synthesis, Mon. Not. Roy. Astron. Soc. 499 (2020) 2803 [2005.03055].

[63] A. da Silva Schneider, E. O'Connor, E. Granqvist, A. Betranhandy and S. M. Couch, Equation of State and Progenitor Dependence of Stellar-mass Black Hole Formation, Astrophys. J. 894 (2020) 4 [2001.10434].

[64] K. Kritos and I. Cholis, Evaluating the merger rate of binary black holes from direct captures and third-body soft interactions using the Milky Way globular clusters, Phys. Rev. D 102 (2020) 083016 [2007.02968].

[65] J. S. Vink, A. de Koter and H. J. G. L. M. Lamers, Mass-loss predictions for o and b stars as a function of metallicity, Astron. Astrophys. 369 (2001) 574 [astro-ph/0101509]. 
[66] G. Graefener and W. R. Hamann, Mass loss from late-type WN stars and its Z-dependence: very massive stars approaching the Eddington limit, Astron. Astrophys. 482 (2008) 945 [0803.0866].

[67] J. S. Vink, L. E. Muijres, B. Anthonisse, A. de Koter, G. Graefener and N. Langer, Wind modelling of very massive stars up to 300 solar masses, Astron. Astrophys. 531 (2011) A132 [1105.0556].

[68] Y. Chen, A. Bressan, L. Girardi, P. Marigo, X. Kong and A. Lanza, PARSEC evolutionary tracks of massive stars up to 350 solar masses at metallicities $0.0001 \leq Z \leq 0.04$, Mon. Not. Roy. Astron. Soc. 452 (2015) 1068 [1506.01681].

[69] H. Sana, S. E. de Mink, A. de Koter, N. Langer, C. J. Evans, M. Gieles et al., Binary interaction dominates the evolution of massive stars, Science 337 (2012) 444 [1207.6397].

[70] M. Moe and R. Di Stefano, Mind Your Ps and Qs: The Interrelation between Period (P) and Mass-ratio (Q) Distributions of Binary Stars, The Astrophysical Journal Supplement Series 230 (2017) [1606.05347].

[71] L. Hernquist, An Analytical Model for Spherical Galaxies and Bulges, Astrophys. J. 356 (1990) 359.

[72] H. Mo, F. C. van den Bosch and S. White, Galaxy Formation and Evolution. Cambridge University Press, May, 2010.

[73] A. V. Kravtsov, The size - virial radius relation of galaxies, Astrophys. J. Lett. 764 (2013) L31 [1212.2980].

[74] P. Behroozi, R. H. Wechsler, A. P. Hearin and C. Conroy, UniverseMachine: The correlation between galaxy growth and dark matter halo assembly from $z=0-10$, Mon. Not. Roy. Astron. Soc. 488 (2019) 3143 [1806. 07893].

[75] H. Mouri and Y. Taniguchi, Runaway merging of black holes: analytical constraint on the timescale, Astrophys. J. Lett. 566 (2002) L17 [astro-ph/0201102].

[76] R. K. Sheth and G. Tormen, Large scale bias and the peak background split, Mon. Not. Roy. Astron. Soc. 308 (1999) 119 [astro-ph/9901122].

[77] W. H. Press and P. Schechter, Formation of galaxies and clusters of galaxies by selfsimilar gravitational condensation, Astrophys. J. 187 (1974) 425.

[78] K. Schmitz, New Sensitivity Curves for Gravitational-Wave Experiments, 2002.04615.

[79] K. Crocker, T. Prestegard, V. Mandic, T. Regimbau, K. Olive and E. Vangioni, Systematic study of the stochastic gravitational-wave background due to stellar core collapse, Phys. Rev. D 95 (2017) 063015 [1701.02638].

[80] A. J. Farmer and E. S. Phinney, The gravitational wave background from cosmological compact binaries, Mon. Not. Roy. Astron. Soc. 346 (2003) 1197 [astro-ph/0304393].

[81] P. J. McMillan, Mass models of the Milky Way, Mon. Not. Roy. Astron. Soc. 414 (2011) 2446 [1102.4340].

[82] V. De Luca, V. Desjacques, G. Franciolini and A. Riotto, The clustering evolution of primordial black holes, JCAP 11 (2020) 028 [2009.04731]. 